\title{
Balance and muscle strength tests in patients with osteoporotic vertebral fractures to develop tailored rehabilitation programs
}

\author{
Ekaterina V Makarova (1), Larisa A Marchenkova (1), Mikhail A Eryomushkin (2), \\ Elena M Styazkina (2) Ekaterina I Chesnikova (2)
}

(1) Somatic rehabilitation, anti-aging and reproductive health department of FSBI "National Medical Research Center of Rehabilitation and Balneology" of Ministry of Health of Russian Federation; (2) Orthopedics, biomechanics, kinesiotherapy and manual therapy department of FSBI 'National Medical Research Center of Rehabilitation and Balneology' of the Ministry of Health of Russian Federation

This article is distributed under the terms of the Creative Commons Attribution Noncommercial License (CC BY-NC 4.0) which permits any noncommercial use, distribution, and reproduction in any medium, provided the original author(s) and source are credited.

\begin{abstract}
Authors perform a cross-sectional study of functional abilities of the patients with osteoporotic vertebral fractures (VFs). Trunk muscle isometric strength and postural balance impairments were estimated. The development of osteoporotic VFs is associated with a significant decrease in strength of all the body muscles, especially the deep spinal stabilization system, i.e trunk extensor (TE), trunk flexsor (TF), where the VFs contribute to inappropriate distribution of back muscle strength with TE:TF ration of 1:1 instead of 3:2, observed both normally and in patients with uncomplicated osteoporosis. There is also a deterioration of stabilometry and functional coordination assessment tests in patients with pathological VFs, that indicate abnormalities of both static and dynamic postural balance. The obtained data should be taken into account when developing rehabilitation programmes for osteoporosis patients who have suffered compression VFs.
\end{abstract}

Key Words: osteoporosis, vertebral fractures, rehabilitation, trunk muscles, balance function.

Eur J Transl Myol 2020; 30 (3): 9236. doi: 10.4081/ejtm.2020.9236

The life expectancy has steadily increased worldwide, accelerating the incidence of diseases associated with older age. One of them is osteoporosis - a systemic skeletal disease characterized by low bone mass and increased fracture risk. ${ }^{1}$ According to the International Osteoporosis Foundation, an osteoporotic fracture occurs every 3 seconds. Worldwide 1 in 3 women and 1 in 5 men will suffer at least one osteoporotic fracture after the age of $50 .^{2}$ Pathological compression vertebral fractures (VFs) are one of the most frequent and severe complications of osteoporosis. In Russia 2 million compression VFs are registered annually in women and 1 million in men. ${ }^{2}$ According to epidemiological studies conducted in different regions of the country, osteoporotic VFs are found in $7.2-12 \%$ of men and $7-$ $16 \%$ of women. ${ }^{2,3}$

VFs in patients with osteoporosis are observed in the lower thoracic and lumbar spine. In most cases they are accompanied by severe back pain syndrome, disturbance of spine static, formation of pathological thoracic hyperkyphosis, restriction of mobility and motor performance. ${ }^{4}$ Those problems lead to dysfunctionality, disability and require adequate physical rehabilitation methods to improve patient's quality of life. According to scientific data, osteoporosis usually associated with muscle weakness and sarcopenia, as a result of involutive processes in older age.3 Hyperkyposis after the VF violates biomechanics of the spine, affects static and dynamic postural balance., ${ }^{3,4}$ As a result, instability during walking and difficulties in body balance keeping in a standing position can lead to falling and new fractures. ${ }^{5}$ In elderly up to $82 \%$ of fractures are associated with falling. ${ }^{6}$ Taking into account those disturbances, we could state that people with osteoporotic VF's experience motor function disorders.

Motor function is the ability to perform a predetermined movement with maximum certainty. The goal of motor function is optimization of the movement performance at the rate of success, precision, and energy consumption reduction. Motor function could be evaluated in terms of muscle strength, endurance and flexibility parameters, walking speed, as well as postural balance function. ${ }^{5}$

Due to the demand for effective rehabilitation programmes for these patients, it is of interest to study 


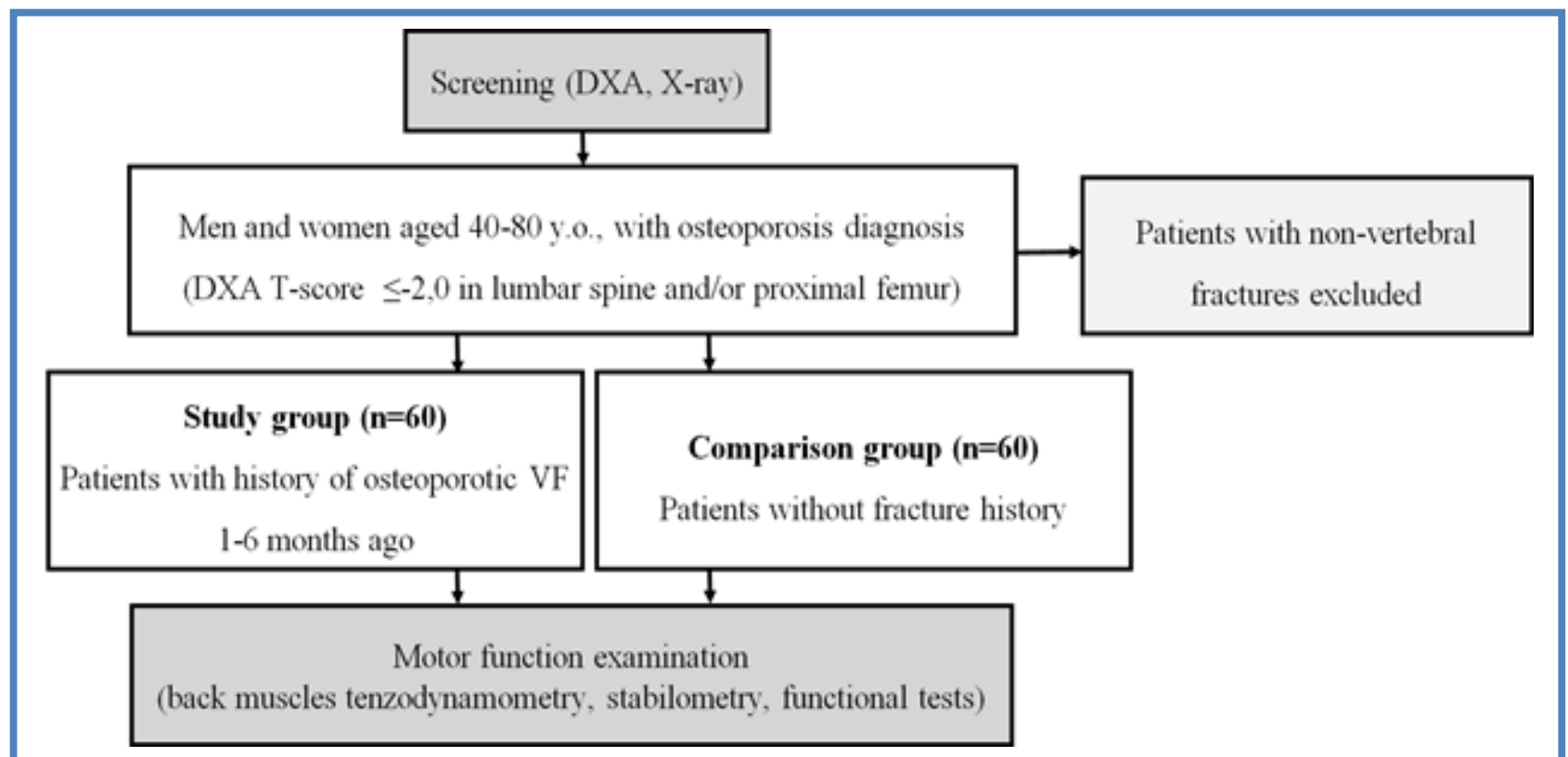

Fig 1. Study design

the peculiarities of motor function in osteoporosis patients who have suffered VFs. There are several researches concerning motor function is individuals with osteoporosis and VFs, ${ }^{3-6}$ but all of them lack global and systematic view of the problem. Thereby all physical rehabilitation programes applied in this patient group in clinical practice lack specificity and efficiency. Understanding pathological processes in musculoskeletal system and disability features in individuals with osteoporotic VFs is a key point for effective rehabilitation programmes development. ${ }^{7,8}$ In our view, an objective assessment of muscle weakness and postural balance function impairments will enrich theoretical base and allow more effective and rational planning of rehabilitation programmes. The scientific hypothesis suggests that such trauma would cause muscle weakness, muscle dysfunction and conditional (basic motor) disturbances.

The objective of this study was to examine muscle strength, motor function and coordination disorders in patients with osteoporotic VFs for better understating of pathological processes and clinical effects in cases of osteoporotic VFs

\section{Materials and Methods}

A cross-sectional study in two groups was conducted on the base of FSBI National Medical Research Centre for Rehabilitation and Balneology' of the Ministry of Health of Russia in 2017-2018. See study design in Figure 1. The study group consisted of 60 patients (4 men and 56 women) aged 40 to 80 years (average age 65,43 $\pm 7,12$ ), who suffered systemic osteoporosis complicated by at least one pathological compression VF not older than 6th months, confirmed radiologically and defined as per
H. Genant criteria,${ }^{9}$ with bone mineral density (BMD) in the lumbar spine or proximal femoral area $\leq-2.0$ ( Tscore) according to dual energy X-ray absorptiometry. ${ }^{1}$ The withdrawal criteria were as follows: refusal to sign an informed consent to participate in a study, cachexia of any caused, illness or drug therapy that negatively affects motor ability and muscle strength.

The control group consisted of 60 patients ( 5 men, 55 women) with osteoporosis, (average age $61,97 \pm 5,22$ ) but without previous history of pathological fractures. The groups were comparable in terms of sex, age, body mass index (BMI), and BMD (T-score in the studied zones $\leq$ 2.0).

The study design did not include any prevention or treatment techniques.

\section{Conformity with the principles of ethics}

The study protocol was approved at a meeting of the local ethics committee of FSBI "National Medical Research Center of Rehabilitation and Balneology" of Ministry of Health of Russia No. 3 of 25.07.2016. All participants of the study signed an informed consent.

\section{Examinations}

The set of examinations in both groups included the following:

1. General clinical examination and history taking.

2. Calculating the absolute 10 -year fracture risk using the international FRAX ${ }^{\circledR}$ tool. $^{2}$

3. Osteodensitometry performed using a Lunar Prodigy dual energy X-ray absorber (produced by General Electric) to determine BMD levels in the lumbar spine (L1-L4) and proximal femur.

4. Strength capacity determined using a Back-Check unit (manufactured by Dr. Wolff, Germany) 
estimating the strength of trunk flexors (TF) muscles, trunk extensor (TE) muscles, left lateral flexors (LLF) and right lateral flexors (RLF), the device registers isometric strength of a certain muscle group. Along with the absolute values of muscle strength in $\mathrm{kg}$, the device determines the degree of muscle strength deficiency in percent, calculated as the difference between the actual values of strength and those recommended for a certain age, sex, height and weight parameters.

5. Study of the coordination function by stabilometry performed through 'Stabilan 01-2' ("PK Ritm", Russia). Computer stabilometry is a method of objective postural balance function assesment. Stabilometric platforms record basic postural characteristics based on measuring the pressure center (PC) coordinates in dynamics. Posture stability is assessed by analyzing the dynamics of load redistribution between the support zones of the heel and toe of each feet during the examination. Statokinesiogram (SCG) is a graphic display of these processes. ${ }^{10,11}$

6. Functional tests for assessing functional capabilities: ${ }^{12-14}$

6.1. "Timed up and go test" (TUG) to assess coordination abilities and endurance. The patient gets up from the chair, walks a predetermined 3 meters distance, turns $180^{\circ}$ and returns to the start position. Norm is up to 10 seconds.

6.2. Static load muscle endurance assessment. For the abdominal muscles - patient is lying on his back, hands on the nape, legs bent in the hip. Trunk is raised and holding position time is recorded. Norm for the 45-60 years old: 40-60 seconds, over 61 years old: up to 40 seconds. For the back muscles - the patient is lying on his stomach, the chest is hanging from the support, the trunk is bent to $30^{\circ}$, hands on the nape, trunk extension is performed, holding position time is recorded. The norm for 45-60 years old: from 60 to 90 seconds, over 61 years old: up to 60 seconds.

6.3. Dynamic load muscle endurance assessment. For the abdominal muscles - patient is lying on his back, legs bent at the knees at $60^{\circ}$, arms on the chest, trunk flexion is performed. Number of flexions in $1 \mathrm{~min}$ is recorded. The norm for 45-60 years old: up to 30 times, over 61 years old: up to 20 times. For the back muscles - the patient is lying on his stomach, the chest hangs from the support, the trunk is bent to $30^{\circ}$, the arms are on the chest, trunk extension is performed. Norm for persons 45-80 years old: up to 30 times, from 61 years old: up to 20 times.

6.4. "10-meter walking test" for physical tolerance assessment. The time required for the patient to cover the distance from the 2 nd to the 8 th meter is recorded. Norms for persons 40-60 years old: 1.31 $-1.43 \mathrm{~m} / \mathrm{s}$.
6.5. Flexibility assessment according to 3-point scale. Trunk extension assessment - lying on the stomach, the subject rises his arms as far as the lumbar region allows. The inner angle formed by the elbow is evaluated. Trunk flexion assessment - the subject is standing trying to reach the tips of the toes with straightened legs. The degree of inclination of the trunk forward is assessed.

6.6. "One-leg standing test" for static postural balance assessment with eyes open and closed. The subject performs a stand on each leg in turn with eyes open, then with closed eyes. Researcher recorders balance maintaining time. With open eyes, the norm for 45-60 years old: 30 seconds, over 61 years old: 20 seconds; with closed eyes, the norm for 45-60 years old: 15 seconds, over 61 years old: 10 seconds).

6.7. "Stepping test" (Fukuda-Unterberger test) to assess dynamic balance. On command, the subject marches in place with a high elevation of the hip up to $45^{\circ}, 72-84$ steps per minute. Normally, after 50 steps, a person turns around his axis by $20-30^{\circ}$. The degree of deviation and the displacement in meters are estimated.

\section{Statistical analyses}

The statistical analysis was performed in Microsoft Statistica 10.0 using parametric and non-parametric methods. The indicators are given as the average and standard deviation $(\mathrm{M} \pm \mathrm{m})$ with regular distribution or as the median and 25th and 75th quartiles (Me [Q1; Q3]) with irregular distribution. For pairwise comparisons of group indicators, Student-t or Mann-Whitney test with Bonferroni correction was used. The Spearman's rankorder correlation or gamma-correlation method was used to identify the relationship between the two indicators for the final values. The significance level for testing of statistical hypothesis was assumed 0.05 .

\section{Results and Discussion}

In the study group $38.3 \%$ of the patients had one pathological VF, $16.6 \%$ had $2 \mathrm{VFs}, 20 \%$ had $3 \mathrm{VFs}$; in $25.1 \%$ of the cases we saw multiple VF's in the thoracic and lumbar spine radiologically confirmed in the amount from 4 to 9 . The groups were similar in age, weight, height, BMI parameters, as well as the BMD of the lumbar spine (for baseline characteristics see Table 1). However, compared to the control group FRAX® calculations, patients in the study group had a higher absolute risk of new fractures, both for all osteoporotic fractures $(\mathrm{p}=0.000)$ and for femoral fractures $(\mathrm{p}=$ $0.000)$. They also demonstrated a lower BMD level in the left femoral neck $(\mathrm{p}=0.001)$.

Patients of the study group with VFs have demonstrated a significant deficiency of trunk flexor (TF) and trunk extensor (TE) strength, with relatively adequate strength of lateral flexors (LLF and RLF). The strength of all the studied muscles in the control group virtually met the 
Balance and muscle strength tests in osteoporotic patients

Eur J Transl Myol 2020; 30 (3): 9236. doi: 10.4081/ejtm.2020.9236

Table 1. Characteristics of Study Groups

\begin{tabular}{|l|c|c|c|}
\hline Paramenters & Study group (n=60) & $\begin{array}{c}\text { Control group } \\
(\mathrm{n}=60)\end{array}$ & $\mathrm{P}$ \\
\hline Age (years) & $65.43 \pm 7.12$ & $61.97 \pm 5.22$ & 0.09 \\
\hline Weight (kg) & $74.8 \pm 14.2$ & $76.23 \pm 11.03$ & 0.10 \\
\hline Height (cm) & $161.3 \pm 16.3$ & $162.9 \pm 14.8$ & 0.12 \\
\hline BMI (kg/m ${ }^{2}$ ) & $26.74 \pm 4.39$ & $28.73 \pm 5.94$ & 0.09 \\
\hline Osteoporosis anamnesis (years) & $3.21 \pm 8.16$ & $2.98 \pm 7.03$ & 0.2 \\
\hline Postmenopause duration (for women) & $15.86 \pm 8.05$ & $14.10 \pm 5.73$ & 0.10 \\
\hline Postmenopause age (for women) & $49.56 \pm 4.49$ & $49.85 \pm 4.48$ & 0.07 \\
\hline Absolute 10-year risk of a major osteoporotic fractures \\
per FRAX® (\%)
\end{tabular}

Note: Student's t-test and Mann-Whitney U-test were used

recommended values; the difference between the recommended and actual values were statistically insignificant (Table 3, Figure 1).
Absolute muscle strength levels (in $\mathrm{kg}$ ) for all set of muscles in the study group were significantly lower than in patients without osteoporotic VFs (Table 2, Figure 2).

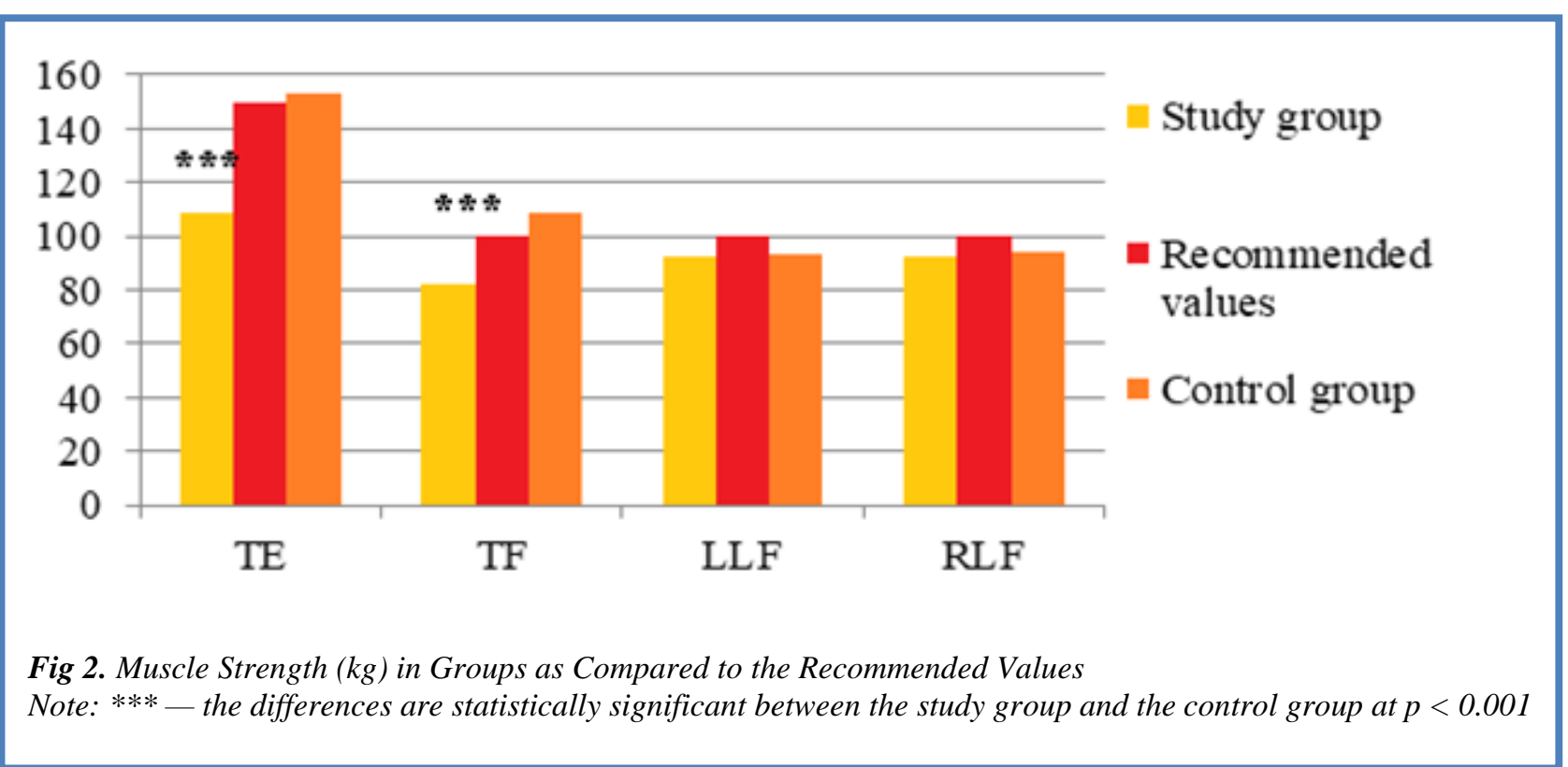


Balance and muscle strength tests in osteoporotic patients

Eur J Transl Myol 2020; 30 (3): 9236. doi: 10.4081/ejtm.2020.9236

Table 2. Levels of Trunk Muscle Strength and Muscle Strength Deficiency Compared to Recommended Values in Patients with Osteoporosis

\begin{tabular}{|l|l|l|l|}
\hline Muscle groups & Study group $(\mathrm{n}=60)$ & Control group (n=60) & $\mathrm{P}$ \\
\hline Trunk extensors $(\mathrm{kg})$ & $15.64 \pm 9.8$ & $27.73 \pm 9.9$ & 0.000 \\
\hline Trunk flexor $(\mathrm{kg})$ & $14.61 \pm 8.98$ & $21.28 \pm 8.38$ & 0.000 \\
\hline Left lateral flexors $(\mathrm{kg})$ & $13.10 \pm 7.2$ & $24.06 \pm 8.9$ & 0.005 \\
\hline Right lateral flexors $(\mathrm{kg})$ & $13.44 \pm 7.43$ & $24.26 \pm 7.65$ & 0.000 \\
\hline
\end{tabular}

Note: Student's t-test was used

Based on the strain-gauge dynamometer measurement data, the following differences in the strength ratio of TE and TF muscles have been found: patients with compression VFs had a relation of TE strength $(15.64 \pm 9.8 \mathrm{~kg})$ to $\mathrm{TF}$ strength $(14.61 \pm 8.98 \mathrm{~kg})$ of approximately $1: 1$, while those without VFs had a ratio of TE strength $(27.73 \pm 9.9 \mathrm{~kg})$ to $\mathrm{FM}$ strength $(21.28 \pm 8.38 \mathrm{~kg})$ of approximately $3: 2$.

According to Table 3, there were no statistically significant differences between the average results of functional tests for assessment of conditional motor abilities obtained in patients of both groups. It should be noted that the results of all four static and dynamic endurance tests in osteoporosis patients of both groups were equally critically low compared to the recommended rates for persons of comparable age [13].

The analysis of the results of the functional OLS test showed that the patients in the study group, compared to the control group, kept poorer postural balance while standing on the left leg with open $(\mathrm{p}=0.05)$ and closed eyes $(\mathrm{p}=0.05)$. When performing the Fukuda stepping test, the patients of the study group showed greater extent of tilting ( $\mathrm{p}=0.02$, Table 4$)$.

According to the stabilometry data (Table 4), the group of patients with pathological VFs was characterised by a lower balance function ratio (BFR) with both open ( $\mathrm{p}=$ $0.000)$ and closed $(p=0.03)$ eyes, as well as larger displacement and deviation in the sagittal plane $(\mathrm{p}=0.01$ and $p=0.025$, respectively). The area of the force plate measurement in both groups was comparable $(p=0.2)$, however, the rate of PC movement appeared to be higher in patients with osteoporosis without fractures in the control group $(\mathrm{p}=0.009)$.

It is known that while aging human organism faces a gradual deterioration of basic motor function due to degenerative changes in musculoskeletal and nervous systems. ${ }^{15,16}$ This is manifested by a significant reduction in endurance, flexibility, strength and muscle volume. ${ }^{17,18}$
A group of Belgian scientists (Beaudart C et al., 2016) has demonstrated that the presence of low BMD and osteoporosis is associated with less skeletal muscle volume and muscle weakness. ${ }^{19}$

Evidence from other studies show lower muscle mass, strength, reduced endurance and flexibility in patients with osteoporotic fractures in the history, compared to the patients of the same age without osteoporosis. ${ }^{20}$ Immobilization or lack of motor performance significantly contributes to muscle dysfunction in such individuals, as involutional processes are rapidly triggered in idle muscles. ${ }^{21}$

For muscle strength assessment in patients with osteoporosis and elderly population doctors and researchers often use functional tests, such as 6 and 10 metre walk tests, endurance tests, TUG test, etc. ${ }^{20}$ While planning the study design, we expand the range of functional tests with coordination tests, objective assessment of postural balance by stabilometry and isometric muscle strength by the means of tenzodynamometery.

The study findings showed that the muscle mass in patients with osteoporotic VFs is generally comparable to that of persons suffering osteoporosis without any fractures. Despite equal muscle mass values, in patients with osteoporotic VFs a significant deficit in back muscles strength of the was revealed $(-40.9 \%$ in TE, $18.1 \%$ in $\mathrm{TF},-8.2 \%$ in LLF, $-8.4 \%$ in RLF) according to tenzodynamometry results). The strength of each muscle group was reduced for $9 \mathrm{~kg}$ an average, compared to individuals without VF. So, patients with osteoporotic VFs demonstrate not quantitative, but qualitative back muscle strength deficiency.

In healthy individuals adequate ratio of TE and TF strength suggests that the extension prevails over flexion. The normal ratio of TE:TF is 3:2. ${ }^{22,23}$ These values were detected in patients without pathological fractures, but patients who experienced osteoporotic VFs a violation of 
Balance and muscle strength tests in osteoporotic patients

Eur J Transl Myol 2020; 30 (3): 9236. doi: 10.4081/ejtm.2020.9236

Table 3. Outcome of Functional Tests for Evaluation of Conditional Abilities in the Concerned Groups

\begin{tabular}{|c|c|c|c|c|}
\hline Tests & Normal rates & $\begin{array}{l}\text { Study group } \\
\qquad(\mathrm{n}=60)\end{array}$ & $\begin{array}{l}\text { Control group } \\
\quad(n=60)\end{array}$ & $\mathrm{P}$ \\
\hline Timed up and go test (s) & up to 10 & $10.0[7.7 ; 13.0]$ & $9.0[8 ; 10]$ & 0.3 \\
\hline $\begin{array}{l}\text { Endurance of back muscles to static } \\
\text { load (s) }\end{array}$ & $60-90$ & $0[0 ; 7]$ & $0[0 ; 30]$ & 0.29 \\
\hline $\begin{array}{l}\text { Endurance of abdominal muscles to } \\
\text { static load (s) }\end{array}$ & $40-60$ & $0[0 ; 11]$ & $0[0 ; 0]$ & 0.18 \\
\hline $\begin{array}{l}\text { Endurance of back muscles to } \\
\text { dynamic load (times) }\end{array}$ & 30 & $0[0 ; 5]$ & $0[0 ; 5]$ & 0.99 \\
\hline $\begin{array}{l}\text { Endurance of abdominal muscles to } \\
\text { dynamic load (times) }\end{array}$ & 30 & $0[0 ; 5]$ & $0[0 ; 6]$ & 0.48 \\
\hline 10 metre walk test $(\mathrm{m} / \mathrm{s})$ & $1.3-1.43$ & $1.6[1.2 ; 2.0]$ & $1.4[1.3 ; 1.7]$ & 0.37 \\
\hline \multicolumn{5}{|l|}{ One-leg standing test } \\
\hline With eyes closed, LL, s & $8-20$ & $2.0[0 ; 3.0]$ & $3.5[3.0 ; 5.0]$ & 0.04 \\
\hline With eyes closed, RL, s & $8-20$ & $2.0[0 ; 3.5]$ & $2.5[0 ; 3.0]$ & 0.5 \\
\hline \multicolumn{5}{|l|}{ Fukuda-Unterberger test } \\
\hline Displacement, metres & $0.2-1$ & $1.0[0.5 ; 1.0]$ & $0.8[0.4 ; 1.0]$ & 0.29 \\
\hline Displacement, degrees & $0-30$ & $40.0[25.0 ; 45.0]$ & $30.0[10.0 ; 45.0]$ & 0.02 \\
\hline
\end{tabular}

Note: Student's t-test and Mann-Whitney U-test were used

strength ratio with insufficient extension function was found. TE:TF muscle strength distribution was nonphysiological and meet 1:1 ratio.

Moreover, a significant inverse correlation between the strength of all the studied back muscles and the number of VFs in anamnesis has been revealed. The most pronounced strength deficiency has been found in the muscles of the deep spinal stabilization system maintaining correct posture, TE and TF. Thus, the development of VFs in the setting of systemic osteoporosis is associated with a significant reduction in the strength of trunk muscles, while there is a pathological weakness of extensors and flexor muscles with insufficient extension function, which obviously cause the thoracic hyperkyphosis formation, impaired posture and restrain physical functioning.

Our data showed that patients with VFs demonstrate abnormalities in postural balance, both static and dynamic, which is evidenced by the stabilometry indicators deterioration and functional postural balance assessment tests. It's important to note, that patients themselves did not report problems with coordination and only a few had a history of falls.

A study by Haines T et al. (2008) shows that women with osteoporosis are generally characterized by weakness of the lower limb muscles, which leads to gait instability. ${ }^{24}$ The authors established that patients with osteoporosis suffer displacement of the PC either sideways or forward; that is, they tend to fall sideways or forward, which causes inadequate weight distribution, poor stability, and loss of balance. ${ }^{25}$ Findings of our study are consistent with those data. ${ }^{25}$

In patients with osteoporotic VFs according to stabilometry unphysiological shift of the PC forward and a slow proprioceptive reaction were stated. Compared to individuals without fractured, patients with VFs showed 
Balance and muscle strength tests in osteoporotic patients

Eur J Transl Myol 2020; 30 (3): 9236. doi: 10.4081/ejtm.2020.9236

Table 4. Coordination Abilities in Patients of the Concerned Groups as per the Stabilometry Data

\begin{tabular}{|l|c|c|c|}
\hline Stabilometric parameters & Study group $(\mathrm{n}=60)$ & Control group $(\mathrm{n}=60)$ & $\mathrm{P}$ \\
\hline BFR, \% (eyes open) & $77.0 \pm 7.61$ & $85.65 \pm 9.38$ & 0.000 \\
\hline BFR, \% (eyes closed) & $67.13 \pm 9.78$ & $73.36 \pm 9.97$ & 0.03 \\
\hline Frontal displacement, mm & $3.7[1.03 ; 6.86]$ & $2.16[1.8 ; 4.46]$ & 0.74 \\
\hline Sagittal displacement, mm & $6.8[3.1 ; 37.7]$ & $4.8[1.8 ; 10.73]$ & 0.01 \\
\hline Deviation in the frontal plane, mm & $1.08[-1.16 ; 1.3]$ & $1.0[-1.4 ; 1.2]$ & 0.67 \\
\hline Deviation in the saggital plane, mm & $1.2[-1.07 ; 1.5]$ & $-1.2[-1.5 ; 1.23]$ & 0.025 \\
\hline Pressure center velocity, mm/s & $9.51 \pm 4.43$ & $7.1 \pm 2.7$ & 0.009 \\
\hline
\end{tabular}

Note: Student's t-test and Mann-Whitney U-test were used

a lower balance function ratio with open $(77.0 \%$ versus $85.6 \%)$ and closed eyes $(67.1 \%$ versus $73.3 \%)$, slower PC movement speed $(9.5 \mathrm{~mm} / \mathrm{sec}$ versus $7.1 \mathrm{~mm} / \mathrm{sec})$. The anteposition of the in patients with VFs may be caused by the formation of pathological thoracic hyperkyphosis. A number of studies suggest that the very presence of kyphosis leads to postural disorders, ${ }^{26}$ ] which worsen with the increase of kyphotic angle. ${ }^{27,28}$ Besides, according to several authors, ${ }^{1}$ deviation of PC may be associated with pronounced weakness of the muscles of the spinal stabilization system.

Concerning functional tests, only coordination tests were specific enough to state the difference in postural balance. Patients with VF $2.5 \mathrm{sec}$ on average earlier lost their balance when standing on one leg in OLS test, and showed bigger deviation (10 o on avarage) in Fukuda test.

No significant differences in the results of other tests were stated. Patients with VFs compared to individuals without osteoporotic fractures had comparable static and dynamic endurance, strength and flexibility of back and abdominal muscles, walking speed, TUG according to functional tests. Both groups showed extremely low values (close to zero) compared to the normative.?

This may indicate either overestimated performance standards for the tests or inadequate general state of training in middle-aged and older population due to insufficient adherence to the culture of physical training in everyday life. We could conclude that functional tests provide less sensitivity and validity (that is, the ability to detect the trait, sign or indicator for which they are designed) in comparison with tenzodynamometry of the trunk muscles. Thus, the development of pathological osteoporotic VFs, as well as the disease itself, does not significantly affect the performance of functional tests, and therefore the latter are not informative enough in assessing specific changes in conditional abilities in the setting of pathological VFs. This is due to the low accuracy of measurements and the presence of a human factor (as the assessment is carried out by a study physician).

The conducted analysis leads to the conclusion that tenzodynamometry of trunk muscles is obviously more preferable in assessing muscle strength, as well as in comparison with more common and simple method of assessing muscle function using a manual dynamometer, which allows to determine only the state of the upper limb muscles, ${ }^{29}$ and the postural balance assessment using stabilometry, OLS and Fukuda tests can be extensively used to assess the risk of falls in patients with VFs. ${ }^{30}$

In general, the detected coordination disorders in patients with VFs indicate a high risk of falls. This is especially significant because patients with pathological VFs have a higher absolute risk of new fractures than patients with uncomplicated osteoporosis (Table 1). The obtained data indicate the need to introduce remedial gymnastics, mechanical and physical therapy aimed at increasing muscle strength, functional capabilities, and improving static and dynamic postural balance in rehabilitation programmes for patients with osteoporotic VFs.

In conclusion, the development of osteoporotic VFs is associated with a significant decrease in isometric strength of all the body muscles for $9 \mathrm{~kg}$ on average, especially the deep spinal stabilisation system (TE, TF), where the VFs contribute to inappropriate distribution of back muscle strength with TE:TF ration of 1:1 instead of $3: 2$, observed both normally and in patients with 
Balance and muscle strength tests in osteoporotic patients

Eur J Transl Myol 2020; 30 (3): 9236. doi: 10.4081/ejtm.2020.9236

uncomplicated osteoporosis. There is also a deterioration of stabilometry and functional postural balance assessment tests in patients with pathological VFs, which indicates abnormalities of both static and dynamic postural balance. Our results should be taken into account when developing rehabilitation programmes for patients with osteoporosis, who have suffered compression VFs.

\section{List of acronyms}

BMD - bone mineral density

BMI - body mass index

FSBI - Federal State Budgetary Institution

LLF - left lateral flexors

PC - pressure center

RLF - right lateral flexors

SCG - Statokinesiogram

TE - trunk extensor

TF - trunk flexors

TUG - Timed up and go test

VFs - vertebral fractures

\section{Authors contributions}

EM, implementation of the clinical trial protocol, a set of materials under study, statistical processing, writing the text of the article; LM, development of research protocol, data analysis, writing the text of the article, formulation of conclusions; ME, scientific consultants; ES and EC, implementation of the clinical trial protocol, data collection.

\section{Acknowledgments}

The Authors thank colleagues of the Department of Neurosciences, University of Padova, Italy and of the A\&C M-C Foundation for Translational Myology, Padova, Italy for discussions and critical readings.

\section{Funding None}

\section{Conflict of Interest}

The authors declare they have no financial, personal, or other conflicts of interest.

\section{Ethical Publication Statement}

We confirm that we have read the Journal's position on issues involved in ethical publication and affirm that this report is consistent with those guidelines.

\section{Corresponding Author}

Ekaterina Makarova, FSBI 'National Medical Research Centre of Rehabilitation and Balneology' of the Ministry of Health of Russian Federation, , Moscow, Novyi Arbat 32, 121099 Russia. Phone +7(915) 111-41-37.

ORCID iD: 0000-0003-3767-8475

Email: baudolinoo@gmail.com

\section{Emails and ORCID iD of Coauthors}

Larisa A Marchenkova: lr-march@rambler.ru ORCID iD: 0000-0003-1886-124X

Mikhail A Eryomushkin: medmassage@mail.ru
ORCID iD: 0000-0002-3452-870

Elena M Styazkina: styazelena@yandex.ru ORCID iD: 0000-0003-4612-511

Ekaterina I Chesnikova: ekaterina-bokova@mail.ru ORCID iD: 0000-0002-2603-6170

\section{References}

1. Melnichenko GA, Belaya ZhE, Rozhinskaya LYa, et al. Russian federal clinical guidelines on the diagnostics, treatment, and prevention of osteoporosis. Problems of Endocrinology. 2017;63(6):392-426. (In Russ.). doi:10.14341/probl2017636392-42

2) Lesnyak O.M., Baranova I.A., Belova K.Yu., Gladkova E.N., Evstigneeva L.P., Ershova O.B., Karonova T.L., Kochish A.Yu., Nikitinskaya O.A., Skripnikova I.A., Toroptsova N.V., Aramisova R.M. Osteoporosis in Russian Federation: Epidemiology, socio-medical and economical aspects (Review). Traumatology and Orthopedics of Russia. 2018;24(1):155-168. (In Russ.) https://doi.org/10.21823/2311-2905-2018-24-1155-168

3) Tarantino U, Iolascon G, Cianferotti L, Masi L, Marcucci G, Giusti F, Marini F, Parri S, Feola M, Rao C, Piccirilli E, Zanetti EB, Cittadini N, Alvaro R, Moretti A, Calafiore D, Toro G, Gimigliano F, Resmini G Brandi ML. Clinical guidelines for the prevention and treatment of osteoporosis: summary statements and recommendations from the Italian Society for Orthopaedics and Traumatology. J Orthop Traumatol. 2017. Vol. 18 (Suppl 1). P. 336.

4) WHO Scientific Group on the Burden of Musculoskeletal Conditions at the Start of the New Millennium. The burden of musculoskeletal conditions at the start of the new millennium. World Health Organ Tech Rep Ser. 2003. No 919.

5) Sparto PJ, Newman AB, Simonsick EM, et al. Contributions to lateral balance control in ambulatory older adults. Aging Clin Exp Res. 2018;30(6):633-641. doi:10.1007/s40520-0170819-3

6) Zampieri S, Mosole S, Löfler S, et al. Physical Exercise in Aging: Nine Weeks of Leg Press or Electrical Stimulation Training in 70 Years Old Sedentary Elderly People. Eur J Transl Myol 2015;25:237-42. doi: 10.4081/ejtm.2015.5374.

7) Cataldo A, Bianco A, Paoli A, et al. Resting sympathovagal balance is related to $10 \mathrm{~km}$ running performance in master endurance athletes. Eur $\mathbf{J}$ Transl Myol 2018; 28:7051. doi: 10.4081/ejtm.2018.7051.

8) Slade SC, David L Carey, Anne-Marie Hill, Meg E Morris. Effects of falls prevention interventions on falls outcomes for hospitalised adults: protocol for a systematic review with meta-analysis. BMJ Open. 2017; 7(11): e017864. doi: 10.1136/bmjopen-2017- 
017864 PMCID: PMC5695509PMID: 29133324

9) Genant HK, Jergas M, Palermo L, Nevitt M, Valentin RS, Black D, Cummings SR. Comparison of semiquantitative visual and quantitative morphometric assessment of prevalent and incident vertebral fractures in osteoporosis The Study of Osteoporotic Fractures Research Group., Journal of Bone and Mineral Research.1996;11:984-996.

10) Kubryak O. V., Grokhovsky S. S., Doborodny A. V. Investigation of human support reactions (posturography, stabilometry) and biological feedback in the STPL program. Moscow: MeraTSP; 2018 (in russ).

11) Pankova I.A., Krivoshey I.V., Kubryak O.V. Measures to improve the management of the rehabilitation process using a stable platform. Kremlin Medicine. Clinical Bulletin. 2017; 2(4):153-156 (in russ.)

12) Eryomushkin M.A. Physical activity and health. From therapeutic exercises to parkour. Moscow: Sport; 2016 (in russ.)

13) Ponomarenko G.N. ed. Physical and rehabilitation medicine. National Guidelines. Moscow: GEOTAR-Media; 2017 (in russ.)

14) Sarabon N, Löfler S, Hosszu G, Hofer C. Mobility Test Protocols for the Elderly: A Methodological Note. Eur J Transl Myol 2015;25:253-6. doi: 10.4081/ejtm.2015.5385.

15) Volpi E, Nazemi R, Fujita S. Muscle tissue changes with aging. Curr Opin Clin Nutr Metab Care. 2004; 7(4): $\quad 405-410$. 10.1097/01.mco.0000134362.76653.b2.

16) Bayramoğlu M, Sözay S, Karataş M, Kilinç S. Relationships between muscle strength and bone mineral density of three body regions in sedentary postmenopausal women. Rheumatol Int. 2005 Sep;25(7):513-7. doi: 10.1007/s00296-004-0475-8

17) Armamento-Villareal R, Aguirre L, Napoli N. Changes in thigh muscle volume predict bone mineral density response to lifestyle therapy in frail, obese older adults. Osteoporos Int. 2014 Feb;25(2):551-8 doi: 10.1007/s00198-013-2450-2.

18) Levinger I., Phu S., Duque, G. Sarcopenia and Osteoporotic Fractures. Clinic Rev Bone Miner Metab. 2016 (14): 38. doi: 10.1007/s12018-0169204-6

19) Beaudart C, McCloskey E, Bruyère O. Sarcopenia in daily practice: assessment and management. BMC Geriatr. 2016 Oct 5;16(1):170. doi: 10.1186/s12877-016-0349-4

20) Cederholm T, Cruz-Jentoft AJ, Maggi S. Sarcopenia and fragility fractures. Eur J Phys Rehabil Med. 2013; 49:111-7. PMID: 23575205

21) Tarantino U, Piccirilli E, Fantini M, Baldi J, Gasbarra
E, Bei R. Sarcopenia and fragility fractures: molecular and clinical evidence of the bone-muscle interaction. J Bone Joint Surg Am. 2015;97(5):429437. doi:10.2106/JBJS.N.00648

22) Verkhoshansky, Yu.V. Fundamentals of special strength training in sports. 3rd ed. Moscow: Soviet Sport; 2013 (in russ.)

23) Mikhayloff P.R., Gromov V.A. The ratio of indicators of the development of muscle strength of the flexors and extensors of the trunk as a factor for preventing pain in the lumbar spine. Extreme human activity 2017;(43)2: 21-24. (in russ.)

24) Zhixiong Zhou, Lu Zheng, Dengyun Wei, Ming Ye, Xun Li. Muscular strength measurements indicate bone mineral density loss in postmenopausal women. Clin Interv Aging. 2013; 8:1451-1459. doi: 10.2147/CIA.S48447

25) Goncalves A, Gentil P, Steele J, et al. Comparison of single- and multi-joint lower body resistance training upon strength increases in recreationally active males and females: a within-participant unilateral training study. Eur J Transl Myol 2019;29:8052. doi: 10.4081/ejtm.2019.8052.

26) Lyons JG, Heeren T, Stuver SO, Fredman L. Assessing the Agreement Between 3-Meter and 6Meter Walk Tests in 136 Community-Dwelling Older Adults. J Aging Health. 2015 Jun; 27(4): 594-605. Published online 2014 Nov 4. doi: 10.1177/0898264314556987

27) McGrath RP, Kraemer WJ, Vincent BM, Hall OT, Peterson MD. Muscle Strength Is Protective Against Osteoporosis in an Ethnically Diverse Sample of Adults. J Strength Cond Res. 2017 Sep;31(9):2586-2589. 10.1519/JSC.0000000000002080.

28) Haines T., Kuys S.S., Morrison G., et al. Balance impairment not predictive of falls in geriatric rehabilitation wards. J. Gerontol. Biol. Sci. Med. Sci. 2008; 63:523-28. Doi: 10.1093/ gerona/63.5.523.

29) Abreu DC, Trevisan DC, Costa GC, Vasconcelos FM, Gomes MM, Carneiro AA. The association between osteoporosis and static balance in elderly women. Osteoporosis international. 2010; (21) 9:1487-1491. doi: 10.1007/s11657-009-0023-y

30) Mendy A., Vieira ER, Albatineh AN, Nnadi AK, Lowry D, Gasana J. Low bone mineral density is associated with balance and hearing impairments. Annals of Epidemiology. 2014;(24) 1: 58-62.

Submissions: July 09, 2020

Revision received: July 30, 2020 Accepted for publication: July 30, 2020 